\title{
Preface to Innovations in High-Entropy Alloys and Bulk Metallic Glasses in Honor of Peter K. Liaw
}

This topical collection is to honor Professor Peter K. Liaw for his significant contributions to materials science and engineering and to The Minerals, Metals \& Materials Society (TMS). It presents work that was originally presented at the symposium Innovations in High-Entropy Alloys and Bulk Metallic Glasses: An SMD \& FMD Symposium in Honor of Peter K. Liaw, held at the TMS 2020 Annual Meeting and Exhibition in San Diego, California, USA. (SMD refers to Structural Materials Division and FMD to Functional Materials Division.) The collection contains articles that were accepted in response to an invitation for manuscripts.

Professor Liaw has demonstrated outstanding academic achievements, endless contributions to education, and dedicated service to TMS. He joined TMS as a member when he was a graduate student at Northwestern University, 40 years ago. In March 2018 he received the TMS Fellow Award; the citation reads, "For seminal contributions to the fundamental understanding of fatigue and fracture behavior in metals and alloys, including advanced structural materials." In this topical collection, the invited articles give a timely review of the present fundamental understanding of the advanced structural materials and the latest research methods.

This collection is comprised of review articles and research articles from leading scientists worldwide in their areas of expertise. The review articles provide readers the most up-to-date, comprehensive information on the high-entropy alloys (HEAs), medium-entropy alloys (MEAs), bulk metallic glasses (BMGs), and advanced characterization together with the most recent machine learning and high-throughput applications regarding scientific fundamentals (e.g., formation, thermodynamics, kinetics, structures, defects, and mechanical properties) and biomedical applications.

The collection includes 16 articles. Seven articles focus on the medium- and high-entropy alloys, three articles focus on amorphous materials, and two articles discuss both the amorphous and the high-entropy alloys. There are three articles mainly for the advanced research methods including high-throughput synthesis, characterization, and in situ neutron diffraction measurements for stress analysis, and there is one article for the brazed joints.

The key features of the review articles are briefly highlighted here. Lee et al. comprehensively summarize Professor Liaw's group and his colleagues' research on BMGs and HEAs. This review article provides an understanding of the current research progression in BMGs and HEAs. In terms of the former, this article reviews the study of shearbanding dynamics and the modeling and analysis of the serrated plastic flow. As for HEAs, several representative state-of-the-art experimental and computational approaches are highlighted. Such fundamental understanding of disorder-induced properties of HEAs is critically important to guide future alloy design to improve the performance. For both the amorphous and HEAs, Yan et al. point out the role of the quantifying the ordering for these materials in the "Order and Disorder in Amorphous and High-Entropy Materials." For the high-entropy alloys, Yan et al. characterize the chemical disorder, whereas amorphous alloys are discussed in terms of the topological disorder. In this article, high-entropy films, high-entropy ceramics, and high-entropy alloys are discussed to compare the hypothesis of the entropy stabilization. Specifically, several novel porous lightweight high-entropy nitrides are reported.

Meanwhile, a concise review on BMGs is provided by Maaß focusing on serrated and non-serrated inhomogeneous flow phenomenon. Four directions of research including (i) the statistical characteristics of plastic fluctuations; (ii) using the effective barrier energy as a descriptor for shear stability; (iii) length scales; and (iv) the structure of shear bands that underlie serrated flow are identified that emerged out of the desire to fundamentally understand the intermittent inhomogeneous flow response. These four research directions gear away from the phenomenological stress-strain behavior, but put the underlying shear defect into focus. Maaß's article summarizes the unsolved problems and future research topics of the BMGs. For the mechanical behaviors of the BMGs, Rezvan et al. report the deformation-mode-sensitive behavior under dynamic loading. For the application of the BMGs, Wang et al. report the potential of the Ti-Cu-Zr-Fe-Sn-Si-Ag-Pd system for biomedical applications because of its modulus, enhanced biocorrosion resistance, the cell adhesion, viability, and proliferation behaviors, which demonstrate good biocompatibility as that of the Ti-6Al-4V alloy.

For the HEAs, Mishra and Nene identify unique aspects of mechanical behavior of metastable transformative highentropy alloys. Lam et al. report the elemental effects on $\mathrm{Mn}$ and $\mathrm{Ge}$ to enhance the mechanical behavior of the Cantor Alloys. They found that substitution of Ge for $\mathrm{Mn}$ increases the elastic moduli of different $\{h k l\}$ orientations of the CoCrFeMnNi-based Cantor Alloys. Their findings indicate that tuning minor elemental compositions results in 
improved strength-ductility combination. The underlying deformation mechanisms of the reported $\mathrm{CoCrFeNiGe}_{0.3}$ were examined by in situ neutron diffraction and analysis of the associated diffraction profiles to reveal the underneath mechanisms. Li et al. apply the machine learning approach to design the heterogeneous grain structures. Chou et al. measure the lattice distortion directly using high annular angle dark field-scanning transmission electron microscopy (HAADF-STEM) by fitting the two-dimensional (2-D) images. The effect of the scanning direction and the accuracy of the HAADF-STEM method were considered to obtain the lattice distortion factors. By controlling cold rolling and annealing temperatures, Xue et al. obtain a heterogeneous microstructure in $\mathrm{Fe}_{20} \mathrm{Co}_{30} \mathrm{Cr}_{25} \mathrm{Ni}_{25} \mathrm{HEA}$ with yield stress of $951 \mathrm{MPa}$, ultimate tensile stress of $1,101 \mathrm{MPa}$, and elongation of 0.189 , and they investigate the tensile deformation, including the lattice strain of the alloy.

Besides HEAs, Wang et al. report the formation and mechanical behavior of a body-centered-cubic medium-entropy alloys (MEAs). Nguyen et al. investigate the superplasticity of high-pressure torsion-processed MEAs. They found superplastic elongation of 505 pct at a temperature of $800{ }^{\circ} \mathrm{C}$ and a low strain rate.

Except the BMGs, HEAs, and MEAs, there are advanced methods reported in this article collection. Zhao et al. report a novel high-throughput micro-synthesis of a combinatorial material library through hot isostatic pressing, which accelerates the screening of promising materials for a variety of engineering applications efficiently and cost-effectively. A Co-Fe-Ni ternary alloy system was selected in Zhao et al.'s study, which demonstrates a new way for screening alloy compositions, microstructures, and properties for materials design. Hoffman and Widom assess the method of the cluster variation to explore solid solutions on body-centered cubic lattices in the context of two specific compounds that exhibit opposite ordering trends. They evaluate the temperature-dependent order parameters, correlations, and entropies. In each case, they obtained the temperature variation of the single-point order parameter as well as a variety of higher-order correlation functions. Lam et al. quantify the phase stress partition in a Fe-based alloy using in situ neutron diffraction measurements. The composite-like deformation behaviors of $\alpha$-Fe and $\mathrm{Fe}_{3} \mathrm{C}$ are revealed simultaneously. Finally, Cai et al. report a complete finite element simulation to investigate the dependence of triaxiality and strength enhancement on geometric and material parameters of triaxial constraint and tensile strength enhancement in brazed joints. Two asymptotic limit solutions based on Bridgman and Xia-Shih solutions have been identified to understand the simulation results, respectively. The critical role of void evolution has been revealed. In addition, ductility of the brazed joint, which has not been fully addressed in literature, is investigated by the Gurson-Tvergaard-Needleman model.

Above all, both this topical collection and the symposium provided a great opportunity for invited and contributed speakers from academia, industry, and governments to discuss the current interest and progress in advanced structural and functional materials. The symposium also provided a mechanism for a group of students, faculty members, researchers, engineers, and administrators to promote idea exchanges and advance the fundamentals and applications of materials science and engineering. The symposium consisted of sessions that emphasized processing, microstructures, and mechanical behavior of BMGs and HEAs, to which Professor Liaw has made great contributions. Other sessions addressed fatigue and fracture behavior, theoretical modeling, and simulations of structural materials. In situ studies of microstructural and mechanical damages during deformation were also included, such as neutron and synchrotron diffraction, thermography, electron microscopy, and acoustic emission.

Publisher's Note Springer Nature remains neutral with regard to jurisdictional claims in published maps and institutional affiliations.

Symposium Organizers: MichaelC. Gao National Energy Technology Laboratory, Albany, OR, USA e-mail:Michael.Gao@netl.doe.gov

E-Wen Huang

National Yang Ming Chiao Tung University, Hsinchu, Taiwan

Yanfei Gao and Hahn Choo

The University of Tennessee, Knoxville, TN, USA

Robert Maaß

University of Illinois at Urbana-Champaign, Urbana-Champaign, IL, USA

Yunfeng Shi

Rensselaer Polytechnic Institute, Troy, NY, USA

Soo Yeol Lee

Chungnam National University, Daejeon, South Korea 
Xie Xie

FCA US LLC, Auburn Hills, MI, USA

Gongyao Wang Globus Medical, Inc., Audubon, PA, USA

Liang Jiang Yantai University, Yantai, China 